\section{Homologous recombination as a mechanism to recognize repetitive DNA sequences in an RNAi pathway}

\author{
Zhenyu Zhang,,3 Shwu-Shin Chang, 1,3 \\ Zhenying Zhang, ${ }^{2}$ Zhihong Xue, ${ }^{1}$ \\ Hanxing Zhang, ${ }^{2}$ Shaojie $\mathrm{Li}^{2}$ and $\mathrm{Yi} \mathrm{Liu}{ }^{1,4}$ \\ ${ }^{1}$ Department of Physiology, The University of Texas \\ Southwestern Medical Center, Dallas, Texas 75390, USA; ${ }^{2}$ State \\ Key Laboratory of Mycology, Institute of Microbiology, Chinese \\ Academy of Sciences, ZhongGuanCun, Beijing 100080, China
}

Quelling is an RNAi-related phenomenon that posttranscriptionally silences repetitive DNA and transposons in Neurospora. We previously identified a type of DNA damage-induced small RNA called qiRNA that originates from ribosomal DNA. To understand how small RNAs are generated from repetitive DNA, we carried out a genetic screen to identify genes required for qiRNA biogenesis. Factors directly involved in homologous recombination $(\mathrm{HR})$ and chromatin remodeling factors required for $\mathrm{HR}$ are essential for qiRNA production. HR is also required for quelling, and quelling is also the result of DNA damage, indicating that quelling and qiRNA production share a common mechanism. Together, our results suggest that DNA damage-triggered HR-based recombination allows the RNAi pathway to recognize repetitive DNA to produce small RNA.

Supplemental material is available for this article.

Received November 2, 2012; revised version accepted December 21, 2012.

RNAi is a mechanism of gene silencing conserved from fungi to mammals (Catalanotto et al. 2006; Buhler and Moazed 2007; Ghildiyal and Zamore 2009). Most of the RNAi-related pathways are initiated by production of dsRNA, which is cleaved by Dicer to generate siRNAs. The siRNAs are loaded into a complex containing an Argonaute (Ago) family protein to mediate gene silencing of homologous RNAs. RNAi is an ancient genome defense mechanism that silences transposons and viral invasion (Sijen and Plasterk 2003; Siomi et al. 2008; Wang et al. 2010; Chang et al. 2012). Consistent with this role of RNAi, a significant portion of eukaryotic small RNAs, including siRNA and the piwi-interacting small RNAs (piRNAs), are produced from repetitive DNA loci in fungi, plants, and animals and target active transposon

[Keywords: RNAi; quelling; qiRNA; homologous recombination; DNA damage; repetitive sequences]

${ }^{3}$ These authors contributed equally to this work.

${ }^{4}$ Corresponding author

E-mail yi.liu@utsouthwestern.edu

Article published online ahead of print. Article and publication date are online at http://www.genesdev.org/cgi/doi/10.1101/gad.209494.112. sequences or their relics (Siomi et al. 2008; Ghildiyal and Zamore 2009). How small RNAs are specifically produced from repetitive DNA loci is not clear.

Quelling in the filamentous fungus Neurospora crassa was one of first known RNAi-related phenomenon (Romano and Macino 1992; Catalanotto et al. 2006; Chang et al. 2012). Quelling is triggered by multiple copies of transgenes and produces transgene-specific siRNA (Catalanotto et al. 2006). In the quelling pathway, QDE-1 (Quelling-Deficient-1) first acts as a DNA-dependent RNA polymerase (DdRP) to produce aberrant RNA (aRNA) from the repetitive transgene loci and then uses its RNAdependent RNA polymerase (RdRP) activity to convert aRNA into dsRNA (Cogoni and Macino 1999a; Lee et al. 2010). This process requires the RecQ DNA helicase QDE-3 and the ssDNA-binding complex replication protein A (RPA) (Cogoni and Macino 1999b; Nolan et al. 2008; Lee et al. 2010). dsRNA is then processed by Dicer proteins to produce siRNA, which is then loaded onto the Argonaute protein QDE-2 to mediate post-transcriptional gene silencing (Maiti et al. 2007). In most Neurospora strains, the ribosomal DNA locus has the only highly repetitive DNA sequences. The potent silencing effect of quelling on repetitive transgenes suggests that it is an anti-transposon response. Consistently, the quelling pathway suppresses the replication of a functional transposon (Nolan et al. 2005).

We previously discovered that DNA damage induces the expression of QDE-2 and a class of small RNAs named qiRNAs for their association with QDE-2 /Cecere and Cogoni 2009; Lee et al. 2009, 2010). qiRNAs originate from the rDNA locus, which contains $\sim 200$ copies of rDNA repeats, and their production depends on QDE-1, QDE-3, Dicers, and RPA. qiRNA levels are very low under normal growth conditions. DNA damage-induced small RNAs were recently also discovered in Arabidopsis, flies, and mammals (Francia et al. 2012; Michalik et al. 2012; Wei et al. 2012), suggesting that DNA damage is a common trigger for small RNA production in eukaryotes.

How does the quelling pathway specifically target repetitive DNA sequences? What is the mechanistic link between quelling and the DNA damage-induced qiRNA production? Here we show that the homologous recombination (HR) process is essential for qiRNA production and quelling. We further show that the qiRNA and the quellinginduced siRNA share the same biogenesis mechanism. Together, our results suggest that Neurospora uses HR to distinguish repetitive DNA loci from the rest of the genome.

\section{Results and Discussion}

A genetic screen identified genes required for $H R$ to be essential for qiRNA production

Treatment of Neurospora with DNA damage agents such as histidine, hydroxyurea (HU), or ethyl methanesulfonate (EMS) induces the production of qiRNA and induction of QDE-2 protein expression (Lee et al. 2009). In addition, the DNA damage-induced QDE-2 expression is abolished in mutants defective for qiRNA production and quelling (Lee et al. 2009, 2010). To identify additional components of qiRNA and quelling pathways, we reasoned that mutants deficient in DNA damage-induced QDE-2 expression would also be defective in qiRNA and 
quelling pathways. Therefore, using the available Neurospora knockout mutants (Colot et al. 2006), we carried out a comprehensive QDE-2 Western blot analysis-based screen to identify mutants with impaired histidine-induced QDE-2 expression. Among the >2000 Neurospora knockout mutants examined, we found that a knockout mutant of rad52 (also called mus-11) was deficient in the histidine-induced QDE-2 expression (Fig. 1A).

RAD52 is an essential component of the eukaryotic HR pathway (Sancar et al. 2004). In addition to RAD52, RAD51 and RAD54 are also essential for HR. Thus, we examined the HU-induced qiRNA production in the rad51 (mei-3), rad52, and rad54 (mus-25) mutants. As shown by the small RNA Northern blot in Figure 1B, HU treatment at $1 \mathrm{mg} / \mathrm{mL}$, a concentration that still permits cell growth, resulted in the induction of qiRNA in a wildtype strain, but such a response was abolished in the qde-3, rad51, rad52, and rad54 mutants. We then examined the expression of the rDNA-specific aRNA (sizes ranging from 0.5 to $2 \mathrm{~kb}$ ), the precursor of qiRNA, and found that the HU-induced aRNA was also abolished in the HR mutants (Fig. 1C). In addition, the production of aRNA in the rad54 mutants could be rescued by a transgene that expresses c-Myc-tagged RAD54 (Supplemental Fig. S1). srs2 (mus-50) encodes a DNA helicase that inhibits the HR process by dislodging RAD51 from the RAD51 nucleoprotein filament (Sung and Klein 2006). As expected, we found that knockout of srs2 in Neurospora resulted in elevated levels of HU-induced qiRNA, aRNA, and QDE-2 (Fig. 1B,C; Supplemental Fig. S2).

Because HR is a part of the DNA repair and recombination processes, we examined the HU-induced qiRNA
A

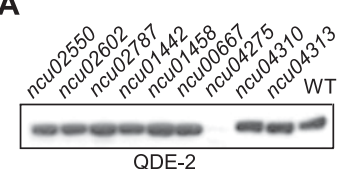

QDE-2

B

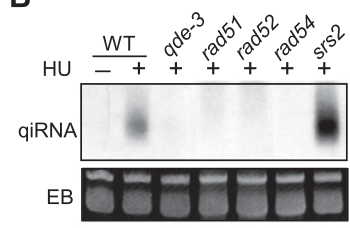

C

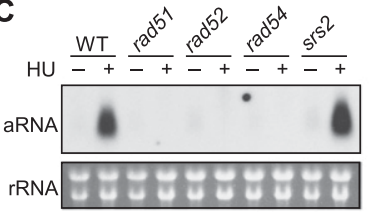

D

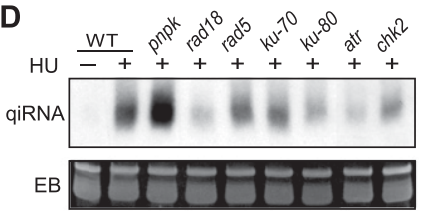

E

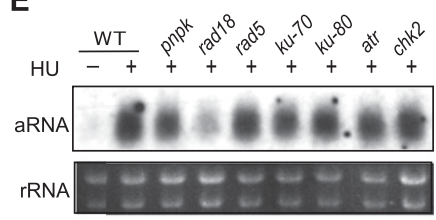

and aRNA production in a series of Neurospora DNA repair and checkpoint pathway mutants (Fig. 1 D,E; data not shown). These mutants included rad18 (post-replication repair), rad5 (post-replication repair), ku70/mus-51 and ku80/mus-52 (nonhomologous end-joining), atr/mus-9 and chk2 (checkpoint), pnpk (single-strand break and base excision repair), and msh3 (mismatch repair). In contrast to the HR mutants, the HU-induced qiRNA and aRNA could still be produced in these mutants. Moreover, the qde-1 and qde-3 mRNA levels in these mutants and in the HR mutants were comparable with those in the wildtype strain, indicating that the phenotype observed in the HR mutants is not due to low QDE-1 or QDE-3 levels (Supplemental Fig. S3). These results indicate that HR, but not other DNA repair and checkpoint pathways, is required for qiRNA synthesis in a step upstream of aRNA production.

\section{Chromatin remodeling factors are required for qiRNA} production due to their roles in $H R$

In our screen, several genes encoding for ATP-dependent chromatin remodeling enzymes were also found to be required for the histidine-induced QDE-2 expression (Fig. 2A; data not shown). These genes encode the Neurospora homologs of swr1, iswi, and chd1 (NCU09993, NCU03875, and NCU03060, respectively). As shown in Figure 2B, both qiRNA and aRNA production induced by HU were completely or mostly abolished in the swr1, iswi, and chd1 mutants, indicating the important roles of these proteins in qiRNA biogenesis. These results further suggest that qiRNA and aRNA production is regulated at the chromatin level.

These chromatin remodeling enzymes use ATP to alter histone-DNA contacts, causing changes in the status of chromatin by moving and restructuring nucleosomes (Clapier and Cairns 2009). Chromatin remodeling factors INO80 and SWR1 have been previously reported to be associated with DNA repair processes, and INO80 is involved in the HR process (Vignali et al. 2000; van Attikum et al. 2007). ISW1 is involved in replication initiation and promotes replication fork progression (Vincent et al. 2008). Because HR is required for qiRNA biogenesis, we hypothesized that these enzymes are also involved in HR. Thus, we examined the recombination rates at the methyltryptophan resistance (mtr) locus in different Neurospora strains by transforming cells with a construct containing the bialaphos resistance gene (bar) that can disrupt the mtr gene by HR (Ishibashi et al. 2006). The targeting of the bar gene into the $m t r$ gene results in transformants that are resistant to both bialaphos and the amino acid analog $p$-fluorophenylalanine (FPA). The wildtype strain has a recombination rate of $\sim 20 \%$ (Fig. 2C), which is a typical HR rate in Neurospora. As expected, the HR rates of the ku80 strain and the rad51 strain are nearly $100 \%$ and $0 \%$, respectively. Consistent with a role of SRS2 in suppressing HR, the srs 2 mutant exhibited an $\sim 50 \%$ HR rate. On the other hand, the HR rates were very low or completely abolished in the $s w r 1$, isw1, and chd1 mutants. These results suggest that these chromatin remodeling enzymes are required for qiRNA production due to their nonredundant roles in HR and that the chromatin remodeling factors act collaboratively to regulate chromatin status during the qiRNA production process. 
A

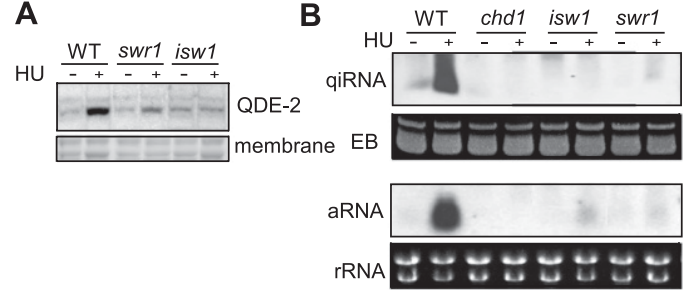

C

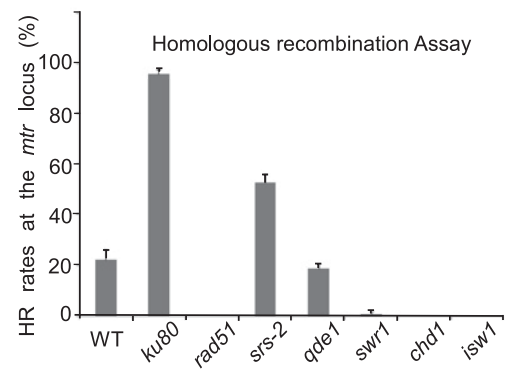

Figure 2. ATP-dependent chromatin remodelers are involved in the qiRNA pathway. (A) QDE-2 Western blot analysis of the indicated strains. Cultures were grown in histidine for 2 d. $(B)$ Northern blot analysis showing qiRNA and aRNA production in different strains after $2 \mathrm{~d}$ of treatment. $(C) \mathrm{HR}$ assays showing the HR rates of the indicated strains. $n=3$; error bar indicates SD.

\section{qiRNA production requires DNA replication}

HR is the predominant DNA double-strand break (DSB) repair pathway during $S$ and G2 phases, when replicated sister chromatin provides a template for HR-dependent repair (Sancar et al. 2004). The accumulation of recombination intermediates correlates with the accumulation of replication intermediates, indicating that HR activity occurs most frequently during DNA synthesis (Zou and Rothstein 1997). To determine whether qiRNA production requires DNA replication, we treated the wild-type Neurospora with different concentrations of HU. At low concentrations of $\mathrm{HU}(0.5$ and $1 \mathrm{mg} / \mathrm{mL})$, qiRNA and aRNA production were induced, whereas at $8 \mathrm{mg} / \mathrm{mL}$ $\mathrm{HU}$, a concentration at which DNA replication is completely blocked (Srivastava et al. 1988), qiRNA and aRNA production were abolished (Fig. 3A,B). Similarly, treatment of wild-type cells with EMS also led to the induction of qiRNA at low concentrations but a blockade of qiRNA synthesis at a high concentration (Fig. 3A). A similar HU dose-dependent response was also observed in an $\mathrm{atm}$ mutant (Fig. 3B). These results suggest that qiRNA biogenesis requires DNA replication even though qiRNA production is induced by modest DNA damage that can result in partial replication inhibition.

To further confirm our conclusion, we created Neurospora strains in which one of the essential components for DNA replication, proliferating cell nuclear antigen (PCNA), can be silenced by quinic acid (QA)-inducible pcna-specific dsRNA (Cheng et al. 2005). PCNA is a processivity factor that forms a complex with DNA polymerase and acts as a clamp that tethers DNA polymerase to the DNA template (Kelman 1997). Figure 3C shows the race tube results that compared the growth phenotypes of the wild-type strain and two independent pcna knockdown strains (dspcna). In the presence of QA, the cell growth of the dspcna strains was dramatically inhibited, indicating that DNA replication was inhibited by the silencing of pcna (Fig. 3C). In the presence of QA, the HU-induced qiRNA production was completely abolished in the dspcna strains (Fig. 3D). To examine whether the inhibition of DNA replication leads to a nonspecific loss of all small RNA, we examined the levels of an siRNA at different concentrations of $\mathrm{HU}$ and found that albino-1 (al-1) siRNA (produced from a double-stranded al-1 hairpin RNA) was expressed at similar levels at all concentrations of HU tested (Fig. 3E). This result suggests that HU treatment does not affect the stability of small RNA. Together, these results suggest that qiRNA biogenesis requires DNA replication and occurs during the $S$ phase of the cell cycle.

\section{rDNA-specific recombination intermediates accumulate upon DNA damage}

The requirements for HR and the RecQ DNA helicase QDE-3 in qiRNA production raised the possibility that recombination of rDNA repeats results in production of an "aberrant" DNA structure that acts as a trigger for qiRNA production. To test this hypothesis, we subjected unsynchronized Neurospora cultures to HU treatment, extracted genomic DNA, and performed two-dimensional (2D) electrophoresis assays. In the absence of $\mathrm{HU}$, little or no replication intermediates of rDNA could be observed (Fig. 4). The HU treatment resulted in the accumulation of rDNA-specific replication intermediates in both the wildtype and srs2 strains, which are represented as an "arc" in the 2D electrophoresis assay (Brewer and Fangman 1987). In addition, a low level of recombination intermediates (Fig. 4, indicated by an open arrow), which are typically represented as a spike above the replication intermediates
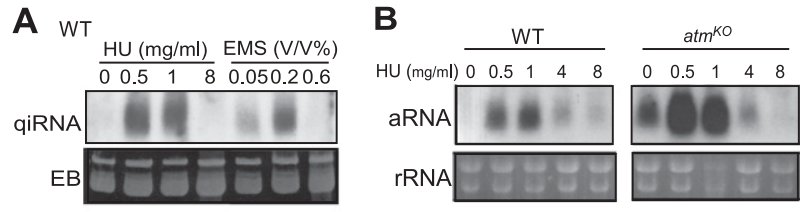

C

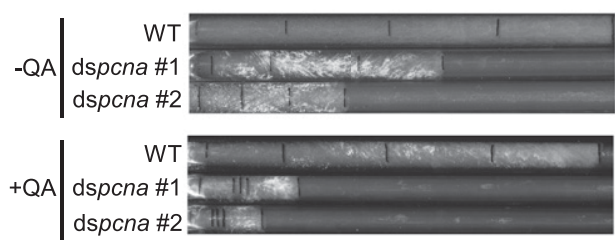

D

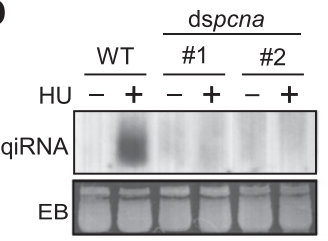

E

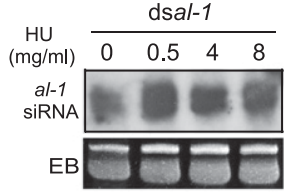

Figure 3. DNA replication is required for qiRNA biogenesis. $(A)$ Northern blot analysis showing the levels of qiRNA in the wildtype strain grown in the indicated concentrations of HU and EMS. (B) Northern blot analysis showing aRNA production in different strains. $(C)$ Race tube analysis showing the growth of the indicated strains in race tubes. dspcna $\# 1$ and \#2 strains are two independent dsRNA knockdown strains in which pcna dsRNA expression can be induced by QA $\left(1 \times 10^{-3} \mathrm{M}\right)$. The black lines indicate cell growth fronts that were marked every $24 \mathrm{~h}$. (D) Northern blot analysis showing elimination of qiRNA production in the dspcna strains. Culture medium contained $10^{-3}$ M QA. (E) Northern blot analysis showing the levels of al-1 siRNA in the dsal-1 strain that expresses al-1-specific dsRNA. 


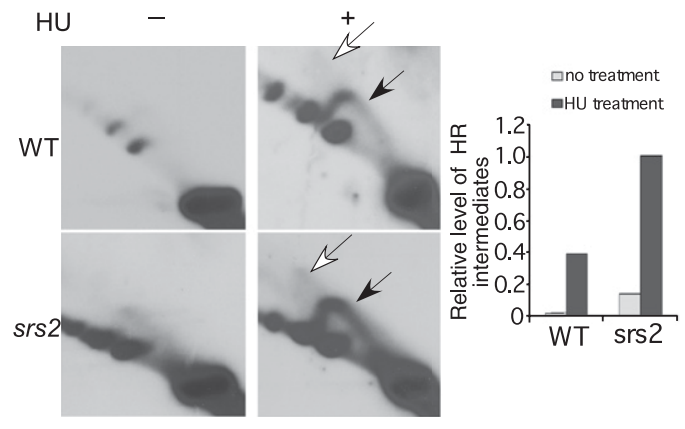

Figure 4. HR intermediates are induced upon DNA damage. 2D gel electrophoresis assay of the wild-type strain and srs2 mutant strain showing the accumulation of replication and HR intermediates upon $\mathrm{HU}$ treatment. HU concentration was $1 \mathrm{mg} / \mathrm{mL}$. (Solid arrows) Replication intermediates; (open arrows) recombination intermediates. The quantification of the 2D gel electrophoresis assay is shown. The relative amounts of total HR intermediates were normalized to the result from the srs2 mutant treated with HU.

on the 2D gels (Brewer and Fangman 1987; Zou and Rothstein 1997), appeared for the HU-treated wild-type culture. Consistent with a negative role for SRS2 in the HR process, the levels of both replication intermediates and recombination intermediates were further increased in the srs 2 mutant. This result suggests that HU treatment results in the formation of recombination-mediated DNA structures, which are likely the trigger for qiRNA production.

\section{Quelling requires $H R$ and is also induced by DNA damage}

Quelling-triggered gene silencing and siRNA production occur under normal growth conditions and do not require DNA damage agent treatment. However, the similarity between quelling and qiRNA biogenesis suggest that these two phenomena may be mechanistically the same. To test this hypothesis, we performed quelling assays by transforming Neurospora with an al-1 transgene. As shown in Figure 5A, 29\% of the wild-type transformants exhibited quelling, as indicated by the change of conidia color from orange to yellow or white. In contrast, very low quelling efficiency was observed in the qde-3, rad51, and rad54 strains, indicating that, like QDE-3, the HR components are required for quelling.

Because repetitive DNA is known to be a major cause of genome instability due to recombination (Bzymek and Lovett 2001; Vader et al. 2011), we hypothesized that quelling is also caused by DNA damage due to repetitive transgenes. Thus, we examined whether the quellingtriggered siRNA, like qiRNA production, is triggered by DNA damage and requires DNA replication. We reasoned that a fully quelled strain might already be subject to high levels of DNA replication stress at the quelled locus and that further DNA damage treatment would not further promote the production of siRNA. Therefore, a partially al-1-quelled wild-type transformant (yellow conidia) was isolated and treated with $\mathrm{HU}$ at different concentrations. As shown in Figure 5B, the level of al-1 siRNA was induced to a level that was similar to that of a fully quelled strain at low concentrations of $\mathrm{HU}$, but the production of siRNA was completely abolished at $4 \mathrm{mg} / \mathrm{mL}$ HU. As expected, $0.5 \mathrm{mg} / \mathrm{mL} \mathrm{HU}$ treatment of the partially quelled strain resulted in a decrease of al-1 mRNA to a level that was comparable with that of the fully quelled strain (Supplemental Fig. S4). These results suggest that the quellingtriggered siRNA production is also the result of DNA damage and requires DNA replication.

To determine whether the HR pathway is directly involved in quelling, we expressed c-Myc-tagged RAD51 in an al-1-quelled strain and performed a chromatin immunoprecipitation (ChIP) assay using a monoclonal c-Myc antibody. As shown in Figure 5C, a significant enrichment of Myc-RAD51 was observed at the al-1 transgene locus. Taken together, these results indicate that quelling and qiRNA biogenesis share the same pathway and that HR is also an essential step in quelling.

\section{Lack of qiRNA production under normal growth conditions is due to mechanisms that prevent rDNA recombination}

Why is qiRNA only induced after DNA damage treatment, whereas the quelling-triggered siRNA is produced under normal growth conditions? The eukaryotic rDNA region consists of several hundred copies of tandem rDNA repeats that each contains a potential origin of DNA replication. Because of its highly repetitive nature, hyperrecombination can occur at a rDNA locus, resulting in genome instability and chromosomal rearrangements. Previous studies have shown that several mechanisms, including rDNA transcriptional silencing and regulation of rDNA replication, prevent rDNA hyperrecombination and maintain rDNA copy numbers (Calzada et al. 2005; Huang et al. 2006). During rDNA replication, the intergenic replication fork barrier sites in rDNA repeats can

\section{A}

\begin{tabular}{|c|c|c|}
\hline & $\begin{array}{c}\text { No Silencing } \\
\text { (Orange) }\end{array}$ & $\begin{array}{c}\% \text { al-1 silencing } \\
\text { (Yellow \& White) }\end{array}$ \\
\hline WT (318) & $71 \%(226)$ & $29 \%(92)$ \\
\hline qde-3 (60) & $96.3 \%(57)$ & $3.8 \%(3)$ \\
\hline rad51 (318) & $99 \%(314)$ & $1 \%(4)$ \\
\hline rad54 (270) & $97 \%(263)$ & $3 \%(7)$ \\
\hline
\end{tabular}

\section{B}
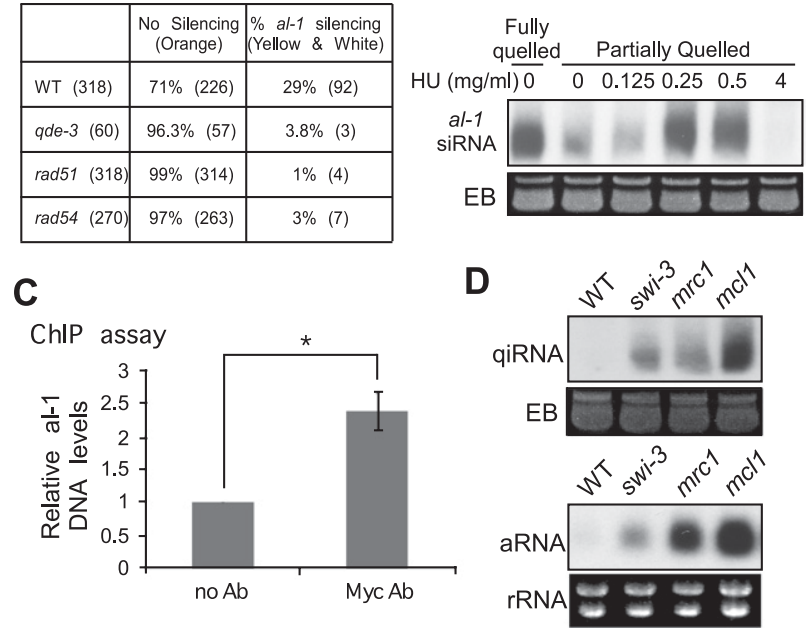

Figure 5. Quelling and qiRNA pathways are mechanistically the same. (A) Quelling efficiency of the indicated strains. The numbers in the parentheses indicate the total number of strains tested, unquelled strains, or quelled strains. $(B)$ Northern blot analysis showing that al-1-specific siRNA was induced by HU treatment in a partially al-1-quelled strain. A fully quelled strain was used as a control. (C) ChIP-qPCR analysis using a monoclonal c-Myc antibody showing the enrichment of Myc-RAD51 at the quelled al-1 locus in a quelled strain expressing Myc-RAD51. $n=4 ;\left(^{\star}\right) P<0.01$; error bars indicate SD. (D) Northern blot analysis showing the levels of qiRNA and aRNA in the indicated mutant strains in the absence of DNA damage treatment. 
stall replication forks unidirectionally so that replication of the rDNA repeats occurs in the same direction as rDNA transcription. The stalled replication forks are protected and maintained by a fork protection complex, which prevents the collapse of the replication fork and is important for maintaining genome stability at the rDNA region. In yeast, the components of this complex have been shown to be important for the maintenance and progression of the replication fork at the rDNA replication barrier sites (Krings and Bastia 2004; Mohanty et al. 2006).

We hypothesized that mechanisms that suppress rDNA recombination during replication inhibit qiRNA production under normal growth conditions. The Neurospora swi3 (NCU01858), mrc1 (NCU04321), and mcl1 (NCU08484) genes encode the homologs of the fission yeast Swi3p, Mrclp, and Mcllp, respectively, which are part of the fork protection complex. To test our hypothesis, we examined the production of qiRNA and aRNA in the swi3, mrc1, and mcl1 mutants. As shown in Figure 5D, the levels of both qiRNA and aRNA were high even in the absence of DNA damage agent treatment in these mutant strains. In the fission yeast, SWI3 is important for the replication fork arrest in the rDNA region, and deletion of swi3 resembles the HU-induced replication stress at the rDNA region (Krings and Bastia 2004). Quelling assay results showed that these replication fork protection mutants have normal quelling efficiency (Supplemental Table S1), suggesting that these factors do not have a major impact on repetitive transgenes. These results suggest that the difference between qiRNA production and quelling is that the rDNA locus is normally actively protected from $\mathrm{HR}$, but the repetitive transgene loci are not; thus, quelling occurs under normal growth conditions.

In this study, we identified $\mathrm{HR}$ as an essential process for the DNA damage-induced qiRNA production and quelling. Because qiRNA and quelling are both produced from repetitive DNA loci and the normally protected rDNA locus is the only highly repetitive DNA locus in the Neurospora genome, our results suggest that HR is a mechanism that can distinguish repetitive foreign DNA from the rest of the genome. Consistent with this conclusion, the quelling pathway has been shown to suppress transposon proliferation in Neurospora (Nolan et al. 2005).

Our study provides important insights into the mechanism of small RNA production from repetitive DNA loci. Even though qiRNA and the quelling-induced siRNA may appear to be triggered by different cues, we showed that the upstream mechanism for their production is the same and that both are results of DNA damage. First, the biogenesis of both types of small RNA require the same set of components, including QDE-1, QDE-3, Dicers, RPA, and the HR components. Second, like qiRNA, the transgene-induced siRNA can also be induced by DNA damage and requires DNA replication. Repetitive DNA is known to be a major source of genome instability in different organisms due to HR (Bzymek and Lovett 2001; Vader et al. 2011). Therefore, even under normal growth conditions, repetitive transgenes lead to DNA replication stress or DSBs, resulting in the production of transgene-specific siRNA. Third, in mutants that are deficient in maintaining fork stability and progression in the rDNA locus, qiRNA levels are high without DNA damage treatment (Fig. 5D). This result suggests that the rDNA locus is normally protected to maintain its stability and suppress DNA recombination.
aRNA is the precursor of dsRNA. The production of aRNA and dsRNA is catalyzed by the DdRP/RdRP QDE-1 (Lee et al. 2010). How does QDE-1 recognize the quelled locus and rDNA? How does DNA damage trigger the production of qiRNA and siRNA? Our genetic screening results indicate that only the HR process, but not other DNA repair or checkpoint pathways, is required for qiRNA production. After DSBs, the Rad51-coated ssDNA, with the help of Rad54, invades the sister chromatin to form recombination intermediates. Even though how repetitive DNA is recognized by the quelling machinery is not clear, our results suggest a model in which DNA damage promotes the formation of "aberrant" forms of HR intermediates of repetitive DNA, which are recognized by QDE-3, the Neurospora homolog of the BLM/Werner helicase. Together with RPA, they recruit QDE-1 to produce aRNA and dsRNA (Lee et al. 2010). Consistent with this model, HU treatment leads to the accumulation of rDNA-specific recombination intermediates. This model provides an explanation of why small RNAs are specifically produced at repetitive DNA loci: Only the repetitive transgenes and rDNA array provide abundant donor sequences for HR. In addition, BLM helicases have been shown to be recruited to DNA damage sites and play an important role in resolving aberrant recombination intermediates (De Muyt et al. 2012).

Repeat-associated small RNAs have been found in almost all eukaryotes. Since our report of qiRNA, DNA damage-induced small RNAs have been demonstrated in Arabidopsis, Drosophila, and mammals (Francia et al. 2012; Michalik et al. 2012; Wei et al. 2012), suggesting that DNA damage is a common trigger for small RNA production in eukaryotes. Because of the conservation of eukaryotic RNAi pathways and the fact that repetitive DNA can be a trigger for DNA damage, our results suggest that $\mathrm{HR}$ is also likely to be involved in small RNA production processes in other organisms.

\section{Materials and methods}

\section{Strains and growth conditions}

A wild-type strain of $N$. crassa (FGSC4200) was used in this study. Mutant Neurospora strains are listed in Supplemental Table S2. The Neurospora knockout mutant strains used in this study were obtained from the Fungal Genetic Stock Center. Liquid cultures were grown in minimal medium

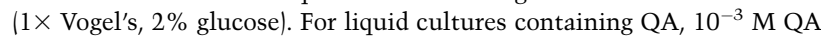
$(\mathrm{pH} 5.8)$ was added to the culture medium containing $1 \times$ Vogel's, $^{\prime} 0.1 \%$ glucose, and $0.17 \%$ arginine. To induce qiRNA production, histidine $(1 \mathrm{mg} / \mathrm{mL})$ or the indicated concentrations of HU were added, and cultures were collected $48 \mathrm{~h}$ later (Lee et al. 2009). For race tube assays containing QA, no glucose was added to the medium.

Quelling assay, RNA Northern blot analyses, HR assay, 2D gel electrophoresis, and ChIP assay

Detailed methods for the quelling assay, RNA Northern blot analyses, HR assay, 2D gel electrophoresis, and ChIP assay are described in the Supplemental Material.

\section{Acknowledgments}

We thank Cuihong Chen and Guojun Wu for assistance in the genetic screen, and Dr. Chul-Hwan Lee for technical support. We thank Yunkun Dang, Liande Li, Qiuying Yang, Haiyan Yuan, and Annie Ye for technical assistance, and Dr. Hirokazu Inoue for providing the bar-containing plasmid for HR assay. This work was supported by grants from the National 
Institutes of Health, the Welch Foundation (I-1560), and the National Natural Science Foundation (31028001) to Y.L.

\section{References}

Brewer BJ, Fangman WL. 1987. The localization of replication origins on ARS plasmids in S. cerevisiae. Cell 51: 463-471.

Buhler M, Moazed D. 2007. Transcription and RNAi in heterochromatic gene silencing. Nat Struct Mol Biol 14: 1041-1048.

Bzymek M, Lovett ST. 2001. Instability of repetitive DNA sequences: The role of replication in multiple mechanisms. Proc Natl Acad Sci 98: 8319-8325.

Calzada A, Hodgson B, Kanemaki M, Bueno A, Labib K. 2005. Molecular anatomy and regulation of a stable replisome at a paused eukaryotic DNA replication fork. Genes Dev 19: 1905-1919.

Catalanotto C, Nolan T, Cogoni C. 2006. Homology effects in Neurospora crassa. FEMS Microbiol Lett 254: 182-189.

Cecere G, Cogoni C. 2009. Quelling targets the rDNA locus and functions in rDNA copy number control. BMC Microbiol 9: 44.

Chang SS, Zhang Z, Liu Y. 2012. RNA interference pathways in fungi: Mechanisms and functions. Annu Rev Microbiol 66: 305-323.

Cheng P, He Q, He Q, Wang L, Liu Y. 2005. Regulation of the Neurospora circadian clock by an RNA helicase. Genes Dev 19: 234-241.

Clapier CR, Cairns BR. 2009. The biology of chromatin remodeling complexes. Annu Rev Biochem 78: 273-304.

Cogoni C, Macino G. 1999a. Gene silencing in Neurospora crassa requires a protein homologous to RNA-dependent RNA polymerase. Nature 399: $166-169$.

Cogoni C, Macino G. 1999b. Posttranscriptional gene silencing in Neurospora by a RecQ DNA helicase. Science 286: 2342-2344.

Colot HV, Park G, Turner GE, Ringelberg C, Crew CM, Litvinkova L, Weiss RL, Borkovich KA, Dunlap JC. 2006. A high-throughput gene knockout procedure for Neurospora reveals functions for multiple transcription factors. Proc Natl Acad Sci 103: 10352-10357.

De Muyt A, Jessop L, Kolar E, Sourirajan A, Chen J, Dayani Y, Lichten M. 2012. BLM helicase ortholog Sgs1 is a central regulator of meiotic recombination intermediate metabolism. Mol Cell 46: 43-53.

Francia S, Michelini F, Saxena A, Tang D, de Hoon M, Anelli V, Mione M, Carninci P, d'Adda di Fagagna F. 2012. Site-specific DICER and DROSHA RNA products control the DNA-damage response. Nature 488: $231-235$.

Ghildiyal M, Zamore PD. 2009. Small silencing RNAs: An expanding universe. Nat Rev Genet 10: 94-108.

Huang J, Brito IL, Villen J, Gygi SP, Amon A, Moazed D. 2006. Inhibition of homologous recombination by a cohesin-associated clamp complex recruited to the rDNA recombination enhancer. Genes Dev 20: 28872901.

Ishibashi K, Suzuki K, Ando Y, Takakura C, Inoue H. 2006. Nonhomologous chromosomal integration of foreign DNA is completely dependent on MUS-53 (human Lig4 homolog) in Neurospora. Proc Natl Acad Sci 103: 14871-14876.

Kelman Z. 1997. PCNA: Structure, functions and interactions. Oncogene 14: 629-640.

Krings G, Bastia D. 2004. swil- and swi3-dependent and independent replication fork arrest at the ribosomal DNA of Schizosaccharomyces pombe. Proc Natl Acad Sci 101: 14085-14090.

Lee HC, Chang SS, Choudhary S, Aalto AP, Maiti M, Bamford DH, Liu Y. 2009. qiRNA is a new type of small interfering RNA induced by DNA damage. Nature 459: 274-277.

Lee HC, Aalto AP, Yang Q, Chang SS, Huang G, Fisher D, Cha J, Poranen MM, Bamford DH, Liu Y. 2010. The DNA/RNA-dependent RNA polymerase QDE-1 generates aberrant RNA and dsRNA for RNAi in a process requiring replication protein A and a DNA helicase. PLOS Biol 8: e1000496.

Maiti M, Lee HC, Liu Y. 2007. QIP, a putative exonuclease, interacts with the Neurospora Argonaute protein and facilitates conversion of duplex siRNA into single strands. Genes Dev 21: 590-600.

Michalik KM, Bottcher R, Forstemann K. 2012. A small RNA response at DNA ends in Drosophila. Nucleic Acids Res 40: 9596-9603.

Mohanty BK, Bairwa NK, Bastia D. 2006. The Tof1p-Csm3p protein complex counteracts the Rrm3p helicase to control replication termination of Saccharomyces cerevisiae. Proc Natl Acad Sci 103: 897-902.
Nolan T, Braccini L, Azzalin G, De Toni A, Macino G, Cogoni C. 2005. The post-transcriptional gene silencing machinery functions independently of DNA methylation to repress a LINE1-like retrotransposon in Neurospora crassa. Nucleic Acids Res 33: 1564-1573.

Nolan T, Cecere G, Mancone C, Alonzi T, Tripodi M, Catalanotto C, Cogoni C. 2008. The RNA-dependent RNA polymerase essential for post-transcriptional gene silencing in Neurospora crassa interacts with replication protein A. Nucleic Acids Res 36: 532-538.

Romano N, Macino G. 1992. Quelling: Transient inactivation of gene expression in Neurospora crassa by transformation with homologous sequences. Mol Microbiol 6: 3343-3353.

Sancar A, Lindsey-Boltz LA, Unsal-Kacmaz K, Linn S. 2004. Molecular mechanisms of mammalian DNA repair and the DNA damage checkpoints. Annu Rev Biochem 73: 39-85.

Sijen T, Plasterk RH. 2003. Transposon silencing in the Caenorhabditis elegans germ line by natural RNAi. Nature 426: 310-314.

Siomi MC, Saito K, Siomi H. 2008. How selfish retrotransposons are silenced in Drosophila germline and somatic cells. FEBS Lett 582: 2473-2478.

Srivastava VK, Pall ML, Schroeder AL. 1988. Deoxyribonucleoside triphosphate pools in Neurospora crassa: Effects of histidine and hydroxyurea. Mutat Res 200: 45-53.

Sung P, Klein H. 2006. Mechanism of homologous recombination: Mediators and helicases take on regulatory functions. Nat Rev Mol Cell Biol 7: 739-750.

Vader G, Blitzblau HG, Tame MA, Falk JE, Curtin L, Hochwagen A. 2011. Protection of repetitive DNA borders from self-induced meiotic instability. Nature 477: 115-119.

van Attikum H, Fritsch O, Gasser SM. 2007. Distinct roles for SWR1 and INO80 chromatin remodeling complexes at chromosomal doublestrand breaks. EMBO J 26: 4113-4125.

Vignali M, Hassan AH, Neely KE, Workman JL. 2000. ATP-dependent chromatin-remodeling complexes. Mol Cell Biol 20: 1899-1910.

Vincent JA, Kwong TJ, Tsukiyama T. 2008. ATP-dependent chromatin remodeling shapes the DNA replication landscape. Nat Struct Mol Biol 15: 477-484.

Wang X, Hsueh YP, Li W, Floyd A, Skalsky R, Heitman J. 2010. Sexinduced silencing defends the genome of Cryptococcus neoformans via RNAi. Genes Dev 24: 2566-2582.

Wei W, Ba Z, Gao M, Wu Y, Ma Y, Amiard S, White CI, Rendtlew Danielsen JM, Yang YG, Qi Y. 2012. A role for small RNAs in DNA double-strand break repair. Cell 149: 101-112.

Zou H, Rothstein R. 1997. Holliday junctions accumulate in replication mutants via a RecA homolog-independent mechanism. Cell 90: 8796. 


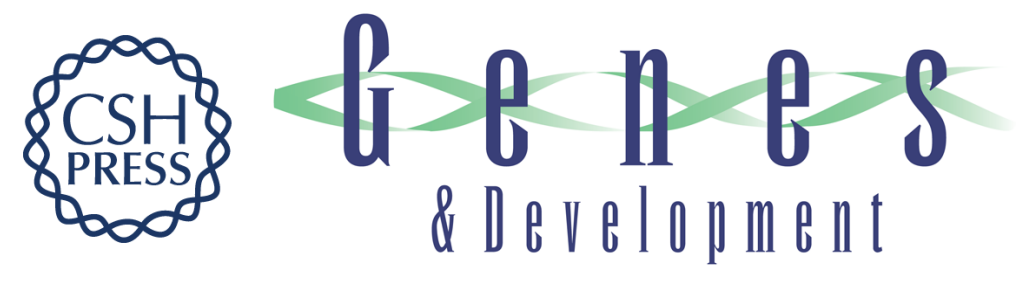

\section{Homologous recombination as a mechanism to recognize repetitive DNA sequences in an RNAi pathway}

Zhenyu Zhang, Shwu-Shin Chang, Zhenying Zhang, et al.

Genes Dev. 2013, 27: originally published online January 15, 2013

Access the most recent version at doi:10.1101/gad.209494.112

\section{Supplemental http://genesdev.cshlp.org/content/suppl/2013/01/09/gad.209494.112.DC1 Material}

References This article cites 39 articles, 12 of which can be accessed free at: http://genesdev.cshlp.org/content/27/2/145.full.html\#ref-list-1

\section{License}

Email Alerting

Receive free email alerts when new articles cite this article - sign up in the box at the top Service

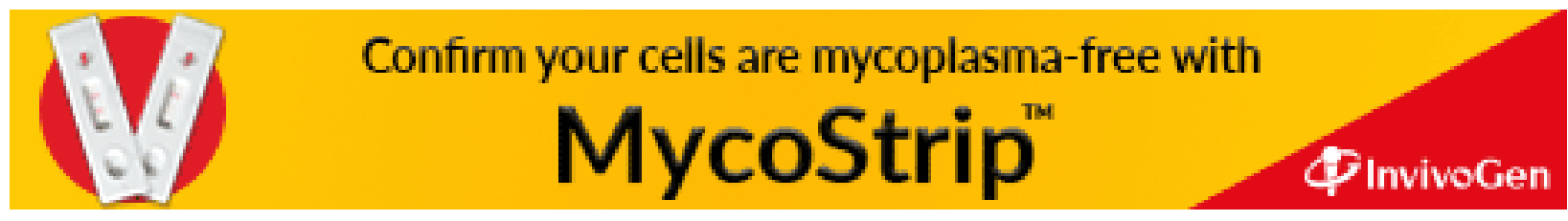

